\title{
Thermo-mechanical Responsive Crystalline Organic Semiconductor
}

Madushani Dharmarwardana, ${ }^{\dagger}$ Gregory T. McCandless, ${ }^{\dagger}$ Raymond P. Welch, ${ }^{\dagger}$ Jeremiah J. Gassensmith $^{\dagger *}$

${ }^{\dagger}$ Department of Chemistry and Biochemistry, University of Texas at Dallas, 800 West Campbell Road, Richardson, TX 75080-3021

Mechanically responsive materials exhibit significant structural changes due to external stimuli such as light, heat and mechanical forces. These materials are widely used as actuators and in energy harvesting applications. Herein, we report that the monoclinic polymorph (M-phase) of the organic semiconductor butoxyphenyl N-substituted naphthalene diimide undergoes clean and complete polymorphic SC-SC phase transition to its highly thermochromic triclinic polymorph (T-phase) upon heating.

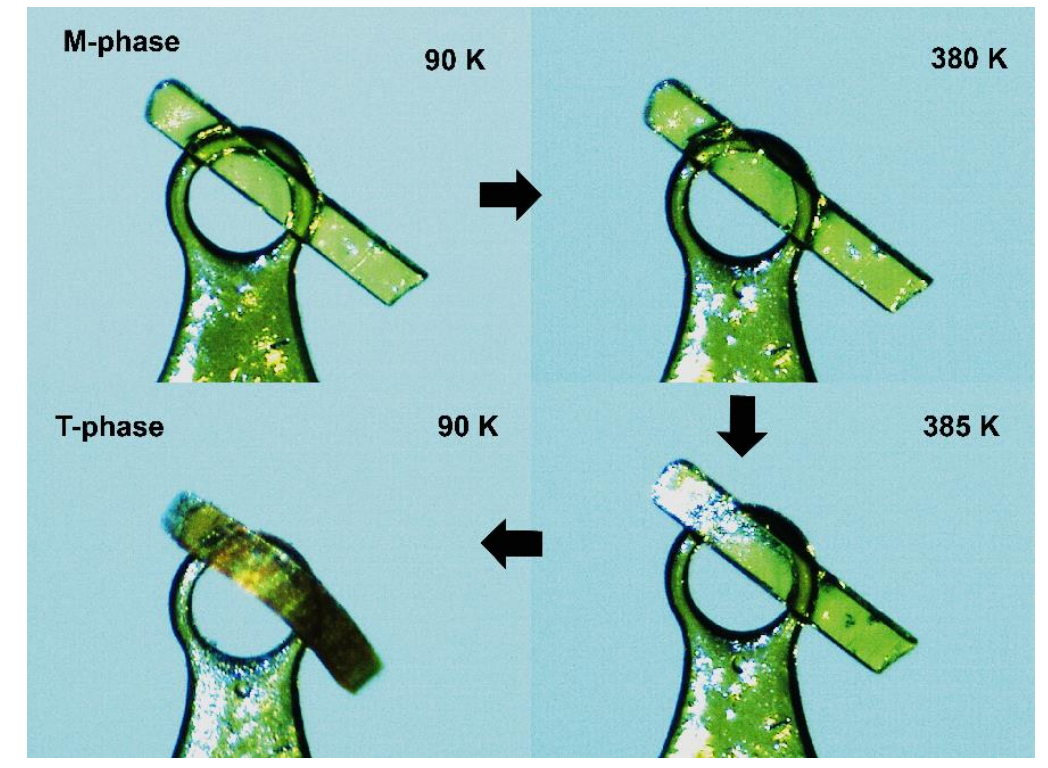

Figure 1. Thermo-mechanical response of single crystals of the M-phase during the phase transition.

More interestingly, heated single crystals of the T-phase show thermo-mechanical responses such as bending and jumping (Figure 1) during the phase transition. While a few reports of thermo-mechanical responsive materials have been published, this phenomenon is quite rare in organic semiconductors such as naphthalene diimides. Upon further examination, we found that when the M-phase was heated beyond the phase transition and cooled down, the typical yellow color of the M-phase crystals has changed to a red/orange color (Figure 1). Temperaturedependent SXRD, temperature-dependent PXRD, and DSC were conducted to confirm that the Mphase has completely transformed to the T-phase. Whereas, optical microscopy was used to analyzed the thermo-mechanical behaviour. 